\title{
Sobre el texto y las fuentes del romance "Mira Nero de Tarpeya"
}

\author{
On text and sources of the romance \\ "Mira Nero de Tarpeya"
}

\author{
Álvaro Alonso \\ Universidad Complutense de Madrid
}

RESUMEN: En este artículo se estudia el romance "Mira Nero de Tarpeya" y la medida en que depende del relato que Tácito y Suetonio hacen del incendio de Roma del 64 d.C. Se señalan luego las posibles relaciones del romance con la tradición medieval de los Mirabilia urbis Romae y sus herederos humanistas, y se propone un paralelismo con el Triunfo púgnico de Vasco Díaz Tanco. En relación con esas cuestiones se discuten las variantes que plantea el verso 10: "Van en Caballo Rotundo, la gente apenas cabía", "Ya en Caballo y en Rotundo, la gente apenas cabía".

Palabras clave: Romancero, humanismo renacentista, mirabilia urbis Romae.

ABSTRACT: This article deals with the romance "Mira Nero de Tarpeya" and its relationship with Tacitus and Svetonius and their description of the fire of Rome in 64. Moreover it studies the possible links between the poem and the mediaeval tradition of Mirabilia urbis Romae and its humanistic heirs. It suggested a parallelism between the poem and the Triunfo púgnico of Vasco Díaz Tanco. Regarding these topics, it discusses the variants of line 10: "Van en Caballo Rotundo, la gente apenas cabía", "Ya en Caballo y en Rotundo, la gente apenas cabía".

Keywords: Romancero, humanism, mirabilia urbis Romae. 


\section{INTRODUCCIÓN}

Comentando el conocido romance "Mira Nero de Tarpeya", Gonzalo Menéndez Pidal (1960: XIII-XIV)recuerda el carácter histórico de los hechos recogidos en el poema (el incendio de Roma del año 64 d.C.), y añade:

Pero para el autor del romance español pasa a segundo plano el episodio del incendio, que acaba por convertirse en un mero pretexto para lucir conocimientos clásicos, y en ello sin duda se cifra hoy para nosotros el encanto del romance: en ese torbellino de alusiones a la historia de Roma.

Matizando esa observación, Giuseppe Di Stefano (1993: 230) ${ }^{1}$ comenta en su edición del romance:

Me resisto a ver en el autor de este $\mathrm{R}$. la víctima de un desmodado afán por lucir erudición. Creo que seríamos nosotros sus víctimas si no captáramos la chispa del juego en sus versos, y no supiéramos apreciar su poemita como regustada caricatura [...] de la erudición [entendida] como desenfrenada danza terminológica y onomástica.

Los dos críticos difieren en su interpretación del poema, perfectamente serio y un poco pedante en la lectura de G. Menéndez Pidal, y cargado de ironía en la de Di Stefano. Ambos comentaristas, sin embargo, coinciden en señalar el despliegue de erudición que hay en esos versos, y apuntan incluso a un recurso estilístico constante en ellos: el "torbellino de alusiones" y "la desenfrenada danza terminológica", es decir, las largas enumeraciones que constituyen uno de los rasgos más característicos del poema.

En las páginas que siguen me propongo analizar esas enumeraciones con objeto de precisar en qué consiste la erudición clásica del autor, y cuáles puedan ser sus posibles fuentes. Daré por supuesto que el romance existía en su versión completa en 1499, si bien en esa fecha La Celestina recoge sólo los dos primeros versos de la composición. Para encontrar el texto íntegro hay que esperar al Cancionero de Velázquez de Ávila, que Rodríguez-Moñino fecha hacia 1535 y que, en todo caso, ha de ser posterior a 1527 (Díaz-Mas, 1985: I, 795-798).

\section{SUETONIO Y TÁCITO}

Dos son las fuentes fundamentales para el incendio del año 64: el libro XV de los Anales de Tácito, y el libro VI, cap. 38, de la Vida de los Césares de

\footnotetext{
${ }^{1}$ Salvo indicación en sentido contrario, todas mis citas del poema proceden de esta edición.
} 
Suetonio. La dependencia del romance con respecto a este segundo relato ha sido ya oportunamente señalada por los editores modernos, pero conviene recordar aquí sus conclusiones (Di Stefano, 1993: 230-232; Díaz-Mas, 1994: 395 $396)^{2}$.

Los versos 1-16 no muestran influencia alguna del historiador latino. El romance describe los gritos y lamentos de los romanos y enumera los lugares por los que pasan en su huida: dos aspectos que faltan en el relato de Suetonio. Los versos 17-24 presentan la destrucción de casas, templos y palacios: un detalle en el que se detiene también la Vida de los Césares, pero sin que haya entre ambos textos correspondencias verbales precisas. En cambio, los versos 25-26 y 32-33 son prácticamente una traducción del historiador latino (Suetonio, 1932: 185):

Por los carneros y ossarios la gente se defendía.

De la Torre de Mecenas [Nerón] lo mirava todo y veía. [...]

De ver abrasar a Roma gran deleite recebía:

vestido en scénico traje decantava en poesía.

Per sex dies septemque noctes ea clade saeuitum est; ad monumentorum bustorumque deuersoria plebe compulsa. Hoc incendium e turre Maecenatiana prospectans, laetusque "flammae — ut aiebat - pulchritudine", Halosin Ilii in illo suo scaenico habitu decantauit.

Entre esas dos reminiscencias tan claras, los versos 28-31 enumeran las más famosas fechorías de Nerón, de las que también da cuenta la obra de Suetonio.

Los versos 34-45 constituyen una larga enumeración de amigos y familiares del emperador que le imploran el perdón de la ciudad. En Suetonio no hay una escena (ni una enumeración) parecida, pero todos y cada uno de los personajes que aparecen en ella están tomados del modelo latino. Es decir, de la fuente proceden los nombres, pero el romance los organiza de acuerdo con su afición por las enumeraciones. Tanto Di Stefano como Díaz-Mas han señalado la imposibilidad de una escena semejante, ya que en ella participan personajes que estaban vivos en los días fatídicos del año 64, como Cecina Tusco (v. 42), junto con otros que ya habían muerto: por ejemplo, Claudia Augusta, niña muerta (a los cuatro meses) un año antes del incendio; Británico, que murió envenenado en el año 55; Octavia, muerta en el 62 (Di Stefano, 1993: 230-232; DíazMas, 1994: 395-396). Estas y otras incongruencias cronológicas no pueden ser un error del autor que, siguiendo a Suetonio, tenía que conocer la fecha de muerte de los personajes que menciona. Se impone, por tanto, la conclusión de que el poeta era indiferente a los anacronismos, e incluso que los buscaba deliberadamente.

\footnotetext{
${ }^{2}$ En lo que sigue adapto mi exposición de "Roma pagana y Roma cristiana: de Nerón al Saco de Roma en la poesía española" (Alonso, 2014).
} 
Mucho menos clara es la influencia de Tácito, XV, 38. Los gritos de la muchedumbre - ausentes, según hemos visto, en el narración de Suetonio- tienen un papel fundamental en los Anales, pero la atención a ese detalle es tan natural en el relato de un incendio, que la coincidencia apenas quiere decir nada. Más llamativa es una coincidencia verbal en la descripción de la huida del pueblo (Tácito, 1925: 487):

Gritos dan niños y viejos y él de nada se dolía.

El grito de las matronas sobre los cielos subía. (vv. 2-3)

Ad hoc lamenta pauentium feminarum, fessa eatate aut rudis pueritiae [...] cunctae impediebant ${ }^{3}$.

Pero también en este caso puede pensarse en una simple coincidencia ya que no es raro, en la descripción de las calamidades públicas, dirigir la atención a ancianos, mujeres y niños.

Al igual que Suetonio, Tácito describe también la ruina de templos, palacios y casas, pero, a diferencia de la Vida de los Césares, ofrece una enumeración completa de los templos destruidos: entre otros, el que Servio Tulio dedicó a Lucina, el que Evandro consagró a Hércules, el de Júpiter Estátor y el palacio de Numa. El romance (vv. 22-23) también ofrece una lista de los templos incendiados, pero ninguno de ellos coincide con los mencionados por Tácito. En fin, en los Anales $(\mathrm{XV}, 44)$ aparece también la idea de que Nerón aprovechó el incendio como pretexto para perseguir a los cristianos.

Algunos indicios, por tanto, sugieren una posible influencia de los Anales, pero ninguno es tan preciso como para dar por seguro ese modelo. En todo caso, desde el punto de vista de sus fuentes antiguas, el romance se divide claramente en dos mitades: hasta el verso 17 (incluso hasta el 24), el poema es independiente de Suetonio y, si acaso, muestra una influencia limitada de Tácito. Desde el verso 17 (quizá el 24) es frecuente, y muy clara, la presencia de la Vida de los Césares.

\section{ENUMERACIONES}

Una vez visto lo que el romance debe a los dos principales modelos latinos, conviene detenerse en sus elementos más originales. Es original la escena en la que los vivos y los muertos suplican a Nerón que se apiade de la ciudad; pero, como ya he apuntado, todos los nombres que figuran en esa enumeración pro-

\footnotetext{
3 José L. Moralejo traduce: "Se añadían, además, los lamentos de las mujeres aterradas, la incapacidad de los viejos y la inexperiencia de los niños” (Tácito, 1980: 238).
} 
ceden de Suetonio. En cambio, ni Suetonio ni Tácito proporcionan los términos de otras tres enumeraciones del romance:

1. La de los lugares por los que huye la muchedumbre (vv. 6-11):

Toda su gente huyendo a las torres se acogía.

Los siete montes romanos lloro y fuego los hundía.

En el grande Capitolio suena muy gran bozería;

por el collado Aventino gran gentío discurría;

van en Cavallo Rotundo, la gente apenas cabía;

por el rico Coliseo gran número se subía.

2. La de los magistrados que lloran la destrucción de la ciudad (vv. 12- 15):

Lloravan los dictadores, los cónsules a porfía,

davan bozes los tribunos, los magistrados plañían,

los qüestores lamentavan, los senadores gemían,

lloran la orden equiestre, toda la cavallería.

3. La de los templos destruidos (vv. 22-23):

El templo capitolino, do Júpiter se servía,

el grande templo de Apolo, el que de Mars se dezía.

\section{EL VERSO 10 Y SUS DOS VARIANTES}

Comenzando por la primera de estas enumeraciones, es preciso detenerse primero en el verso 10, que presenta en las fuentes antiguas dos variantes de interés. El pliego suelto de Praga (Menéndez Pidal, 1960: II, 293-294), el Cancionero de Amberes (Menéndez Pidal, 1945: 213v) y la Silva de romances (Rodríguez Moñino, 1970: 200) leen: "Van en Cavallo Rotundo, la gente apenas cabía".

Siguiendo a la Silva, Di Stefano, en su edición de 1973 (328), imprime "van en Caballo Rotundo" y anota "Caballo rotundo: Circo Máximo4". Por su parte, Díaz-Mas edita también "Caballo Rotundo" y aclara "Se refiere al Circo Máximo". Ninguno de los dos editores señala de dónde procede la identificación "Caballo Rotundo = Circo Máximo", por lo que resulta imposible comparar el verso con su fuente.

El Cancionero gótico de Velázquez de Ávila (Rodríguez-Moñino, 1951: 99102) imprime el verso de manera diferente: "ya en Caualo y en Rotundo la gente apenas cabía".

\footnotetext{
${ }^{4}$ La lectura se mantiene (salvo un retoque ortográfico), pero falta la nota, en la edición que estoy siguiendo, la de 1993.
} 
Acogen esa lectura, con una pequeña alteración ("y en Caualo y en Rotundo”), Agustín Durán (1945: 322), Gonzalo Menéndez Pidal (1960: 6-8) y García de Enterría (1987: 60). Los editores no anotan el verso, que resulta, en efecto, bastante oscuro. Y, sin embargo, la variante de Velázquez de Ávila me parece al menos tan plausible como la otra. "Cavalo" bien pudiera ser "Monte Cavallo", es decir, el Quirinal o, más exactamente, una de sus partes. El término aparecía ya en la novela de caballerías La corónica de Adramón (Anderson 1992: I, li; II, 543), anterior, probablemente, a 1492: "Los rromanos pensavan el de Traetyber que fuese de Nagón; el de Nagón que fuese de Monte Cavallo; y asy pensavan todos".

En cuanto a "Rotundo", podría hacer referencia a Santo Stefano Rotondo, pero me inclino a pensar que el masculino se explica por una atracción de "Cavalo", y que la variante original debía de decir "Rotunda": es decir, Santa María Rotonda, el Panteón. Así aparece, por ejemplo, en las Batallas y quinquagenas de Fernández de Oviedo (1989: 46), no muy lejos, precisamente, de Monte Cavallo. Describiendo una medalla en la que don Alonso de Aragón mandó representar la ciudad de Roma, dice:

En vna lámina de oro [...] estaua Rroma de medio rrelieue muy al proprio contahecha. Allí viérades el castillo de Sanct Angel [...] Sancta María la Redonda [...] e Monte Cauallo e Trastache, e el Coliseo [...] e el Capitolio e otras muchas señas que tiene Rroma.

Fernández de Oviedo ofrece los dos nombres en su forma más completa, pero la forma abreviada del romance no debería causar ningún problema. De hecho, "Cavallo" se atestigua al menos desde el siglo XV, en textos como el anónimo Tractatus de rebus antiquis et situ Urbis Romae: "Palatium Scipionis fuit in caballo, in Via Cornelia", o en el nombre de algunas iglesias, como Sant' Agata in Cavallo o Sant' Andrea in Cavallo (Valentini-Zucchetti, 1953: 127 y mapa final).

Así pues, la variante del Cancionero de Velázquez de Ávila tiene pleno sentido, y debe ser tomada en consideración a la hora de analizar el poema.

\section{MIRABILIA MEDIEVALES Y ARQUEOLOGÍA HUMANÍSTICA}

Conviene pasar ahora al siguiente verso, el 11, y a su referencia al Coliseo. Si se buscan en CORDE (Real Academia Española) Coliseo las referencias al famoso anfiteatro entre 1400 y 1520 , se encuentran ocho citas. Una corresponde a la traducción castellana del De mulieribus de Boccaccio, tres a las Andanzas y viajes de Pero Tafur, tres a Torres Naharro y una a la Carajicomedia. Lo primero que llama la atención son las ausencias: ni Enrique de Villena, ni el 
marqués de Santillana, ni Juan de Mena mencionan el edificio, a pesar de su afición a acumular nombres ilustres relacionados con la Antigüedad. De las ocho menciones que recoge el corpus de la Academia, una corresponde a una traducción del italiano, y nada menos que seis proceden de autores (Tafur y Torres Naharro) que tenían una experiencia directa de la ciudad. Del autor de la Carajicomedia no sabemos casi nada, pero no puede descartarse que también él hubiera estado en Roma. De lo que no cabe duda es de que su referencia al Coliseo funciona en un juego de palabras burlesco (culo, Culiseo) de origen italiano. Es cierto que los datos de CORDE deben tomarse con mucha cautela, pero no puede ser casual que esas ocho menciones se relacionen todas de manera tan estrecha con Italia.

Así que las referencias a Roma que contiene el romance no pueden considerarse tópicas: ni el Coliseo, ni mucho menos Santa María Rotonda o Monte Cavallo formaban parte de la cultura habitual en un escritor de la segunda mitad del siglo $\mathrm{XV}^{5}$. La conclusión que se impone es que el poeta se había documentado, de manera muy deliberada, sobre los monumentos y la topografía de la ciudad. Naturalmente, no puede descartarse que el autor del romance hubiera espigado sus conocimientos de aquí y de allá, pero parece mucho más probable que manejara una fuente que reuniera todas esas informaciones. Lo más natural es suponer que utilizó alguna de las guías de Roma que circulaban desde la Edad Media.

Ya en el siglo XII, un autor anónimo (muy probablemente, un cierto Benedetto di Sancto Pietro) escribió unos Mirabilia urbis Romae, es decir, una suerte de guía de peregrinos que incorpora leyendas, datos de historia antigua e información sobre los principales monumentos de la ciudad, con especial atención a los cristianos, pero sin omitir a los antiguos. A partir de ahí se generó una amplia tradición medieval de reelaboraciones y textos más o menos originales, que ejerció una notable influencia incluso entre los humanistas. No obstante, a lo largo del siglo XV se fue perfilando una aproximación diferente al pasado de la ciudad y su topografía. El interés fundamentalmente religioso de la tradición medieval se vio poco a poco desplazado por una atención creciente hacia los restos arqueológicos y la reconstrucción del mundo antiguo (Murray, 1972: 1-4; Pazienti, 2013). Aunque no prescinden por completo de las iglesias y los monumentos cristianos, tienen ya una clara orientación arqueológica las páginas iniciales del De varietate Fortunae de Poggio Bracciolini y, desde luego, el texto fundamental sobre el tema en los años centrales del Cuatrocientos, la Roma instaurata de Flavio Biondo. Ligeramente anterior a nuestro romance es el De antiquitatibus urbis Romae de Pomponio Leto, y ligeramente poste-

\footnotetext{
${ }^{5}$ Menos convencional todavía sería "Cavallo Rotundo", que no consigo documentar en ningún texto.
} 
rior, de los primeros años del siglo XVI, el Opusculum de mirabilibus novae et veteris urbis Romae de Francesco Albertini (Valentini-Zucchetti, 1953). En España, a finales del XV, Martín Martínez de Ampiés traduce el Viaje de la Tierra Santa de Bernardo de Breyderbach y le antepone un Tratado de Roma, claramente inspirado en los mirabilia medievales (Breyderbach, 1498 y 2003 [1498]).

Es en toda esa tradición, bien en su versión medieval, bien en la humanística, donde el autor del romance pudo encontrar los nombres del Coliseo, del "collado Aventino" y de Santa María Rotonda. Para este último es claro que no pudo valerse de una fuente clásica y, en cambio, es característico de las guías de la ciudad señalar la correspondencia entre los antiguos edificios, o sus ruinas, y la Roma moderna: "in Circo Maximo, ubi nunc orti sunt", "Panthei mutatio in Sanctae Mariae Rotundae ecclesiam", "Ubi nunc est ecclesia Sanctae Mariae Rotundae, ibi fuit Pantheon" (Bracciolini, 1993: 96-97; Biondo, 20052012: II, 193; Leto, 1521: 74r).

Algo diferente es el caso de Monte Cavallo. La mayor parte de los mirabilia y los textos humanísticos dedican una especial atención al Quirinal, pero lo designan casi siempre de esa forma, con su nombre clásico y no con el otro. "Monte Cavallo" no aparece ni en los primitivos Mirabilia, ni en Poggio Bracciolini, ni en las Antiquitates de Pomponio Leto, ni en Martínez de Ampiés. Y cuando la palabra aparece, lo hace de manera episódica, sin que se le dedique un capítulo o un apartado. Así, Flavio Biondo menciona el término una sola vez, y lo hace de pasada, para indicar más claramente la localización de las Exquiliae, que describe en relación con el "montem cui Caballo est nunc appellatio" (Biondo, 2005-2012: I, 111). Otro de los pocos textos que recurren al nombre es el del peregrino alemán del siglo XV Nicolás Muffel, que describe cuatro hermosas estatuas "en Monte Cavallo", "auf dem Rossberg" (Valentini-Zucchetti, 1953: 369). Según explican Valentini-Zucchetti, su obra es una de las menos librescas o, lo que es lo mismo, una de las más próximas a la experiencia real de un visitante de Roma en la Edad Media. Así que, aunque esporádicamente presente en las guías, el término parece más propio de la lengua coloquial, y no puede excluirse que, junto a sus fuentes escritas, el poeta se basara en otras de naturaleza oral o, incluso, en un conocimiento directo de los lugares que cita.

La tercera enumeración, la de los textos destruidos por el incendio, es mucho más convencional: incluye una mención al conocidísimo templo de Júpiter Capitolino y otras dos a "el grande templo de Apolo, el que de Mars se dezía". El autor pudo recordar vagamente algún texto clásico donde se mencinaran templos de Apolo y Marte, sin tener una idea muy clara de su emplazamiento. Pero es posible también que pensara en templos y ubicaciones concretas. Para el de Apolo, por ejemplo, es muy frecuente que tanto los textos medievales como los humanísticos señalen uno en el Vaticano: desde el anónimo Tractatus de rebus 
antiquis, "templum Apollinis qui hodie Sancta Petronilla dicitur" (ValentiniZucchetti, 1953: 133) hasta Albertini, "templum Apollinis in Vaticano". El propio Albertini señala un templo de Marte también en el Vaticano y otro en el Capitolio (Murray, 1972: M4r y N1v).

\section{DE MAGISTRATIBUS}

La segunda lista, la de magistrados que lloran la destrucción de la ciudad, nos lleva al mismo ámbito cultural. Aun más que en el caso de los edificios, me parece improbable que el autor del romance elaborara esa lista sobre la base de una indagación directa en los textos clásicos, así que debió de recurrir a alguno de los tratados sobre el tema que empiezan a escribirse desde los albores del humanismo. En España, ya el marqués de Santillana tenía en su biblioteca un Tratado de los oficios de Roma, además de una copia de las consideraciones de Pier Candido Decembrio sobre "De muneribus Romanae reipublicae" (Gómez Moreno, 1994: 254). El interés por el tema iba frecuentemente asociado a las preocupaciones arqueológicas y topográficas sobre la antigua Roma. Así, Pomponio Leto, a quien ya me he referido como autor de un tratado De antiquitatibus, escribió también un breve tratado De Romanorum magistratibus, sacerdotis, iurisperitis et legibus. De manera que el autor del romance pudo valerse de la tradición de los mirabilia para su primera (y quizá su tercera) enumeración, y de la de los tratados de magistratibus para la segunda. Pero hay otra hipótesis más probable. La idea de ofrecer en un mismo poema ambas enumeraciones bien puede proceder de una estructura frecuente en las guías de Roma, que muchas veces alternan las consideraciones de tipo arqueológico con otras sobre las antiguas magistraturas (Gómez Moreno, 1994: 253). Esa convergencia no puede sorprender: los mirabilia medievales y, más todavía, sus herederos humanísticos quieren dar una idea de la antigua grandeza de Roma. Grandeza que se expresa en la magnificencia de sus edificios y en la sabiduría de sus instituciones.

De manera que el Mirabilia del siglo XII ofrece, en sus capítulos 12 y 14, información sobre los antiguos monumentos y estatuas de la ciudad: "Quare facti sunt caballi marmorei" y "De columpna Antonini et Traiani". Pero entre uno y otro, el capítulo 13 habla "De nominibus iudicum et eorum instructionibus" (Tena Tena, 1999). La tradición no se interrumpe. En la primera mitad del siglo XV, Nicolò Signorili, en su Descriptio, comienza hablando de las murallas de Roma, de las siete colinas de la ciudad, de sus puertas y palacios, pero se interrumpe para hacer un elogio de la Urbe y de sus magistrados, que enumera minuciosamente: reyes, senadores, patricios, cónsules, emperadores, procónsules, decémviros... (Valentini-Zucchetti, 1953: 166). Y ya en el siglo XVI, Alber- 
tini sigue respetando el mismo esquema: después de dedicar el primer libro de su Opusculum a diversos monumentos de Roma, comienza el segundo con un capítulo "De sacerdotibus et magistratibus", aunque vuelve enseguida a "De templis urbis" y "De bibliothecis et basilicis" (Murray, 1972). Así que una sólida convención, que se respetó durante más de tres siglos, llevaba a interrumpir las descripciones de monumentos de la antigua Roma con consideraciones o meras listas de sus instituciones y sus magistrados, de manera muy semejante a como lo hace el romance.

\section{TIEMPO Y ESPACIO ARBITRARIOS}

La tradición de los Mirabilia y sus herederos humanistas nos ayuda a comprender en qué medida era o no "científica" la visión de la ciudad que tenía el autor del romance. Porque lo importante no es la distancia que hay entre lo que dicen los versos y lo que hoy sabemos sobre la antigua Roma, sino la que media entre esos versos y lo que se sabía en su época.

Es indudable que la referencia al Coliseo es anacrónica, ya que el famoso anfiteatro fue construido unos cuantos años después de la muerte de Nerón. Pero ese anacronismo ¿lo era también para los lectores de la época y para el propio autor? La respuesta tiene que ser claramente afirmativa, ya que prácticamente todos los textos señalan que lo que el pueblo llama "Coliseo" se identifica con el anfiteatro de Vespasiano y dejan constancia, por tanto, de su posterioridad con respecto a Nerón (Biondo, 2005-2012: II, 147). Un autor que conocía los tratados sobre magistraturas romanas, que había oído hablar de Monte Cavallo y del propio Coliseo difícilmente podía ignorar la fecha en la que se construyó el anfiteatro. De manera que el anacronismo es aquí tan deliberado como en la enumeración de los cortesanos, vivos y muertos, que suplican a Nerón, o como en la mención de una iglesia cristiana, Santa María Rotonda, en el contexto pagano del poema.

La indiferencia del autor hacia los datos históricos y arqueológicos afecta también a la referencia más famosa de todo el texto, la del verso inicial "Mira Nero de Tarpeya". Como se sabe, arx Tarpeia o sedes Tarpeia es el nombre que se daba a una de las dos cimas del Capitolio. En algunos contextos podía designar al monte con sus dos cumbres, o la ciudadela que había sobre una de ellas.También se empleaba la expresión Mons Tarpeius y, de hecho, una variante del romance que aparece en el manuscrito de Palacio de La Celestina presenta la forma masculina: "Mira Nero de Torpeo" (Di Camillo, 2010: 121). En cualquier caso, ninguna de las dos fuentes clásicas para el incendio del 64 sitúa a Nerón en ese observatorio: Tácito (Anales XV, 39) precisa que Nerón vio arder la ciudad desde su palacio, y Suetonio, como ya se ha visto, sitúa su 
atalaya en lo alto de la Torre de Mecenas. Esta última información es recogida por el poeta en el v. 26, así que, además de una discrepancia con respecto a las fuentes, hay aquí una clara contradicción interna, a no ser que el poeta pensara que la Torre de Mecenas se localizaba en la Roca Tarpeya. Pero los textos arqueológicos de los que podía disponer no autorizaban esa localización. Flavio Biondo recoge una tradición oral, viva todavía en su tiempo, de acuerdo con la cual Nerón vio el incendio desde lo alto de una torre, la Torre Mesa o Torre de Nerón, situada en el Quirinal. "Ninguna mujercilla del barrio —explica Biondo (2005-2012: I, 121) — dejará de contaros que Nerón, divertido y feliz, vio arder la ciudad desde esa torre". Asociando esa tradición con el texto de Suetonio, Biondo identificaba la Torre Mesa con la de Mecenas y, por consiguiente, ubicaba esta última en el Mons Quirinalis, muy lejos de la Roca Tarpeya del romance. Frente a esta localización, que es la más frecuente, Pomponio Leto (1521: 77v) situaba la Torre de Mecenas cerca de la Puerta del Viminal, muy lejos también del arx Tarpeia. En cuanto al palacio de Nerón, algunos, como el anónimo Tractatus, lo colocaban en el Vaticano, otros, como Giovanni Tortelli, cerca de las Exquiliae (Valentini-Zucchetti, 1953: 133; Tortelli, 1495: u6r, s. v. Rhoma): dos lugares que tampoco guardan relación alguna con la localización del romance.

Por tanto, el verso inicial del poema ideaba una escena, la de Nerón en la Roca Tarpeya, totalmente arbitraria desde el punto de vista de los conocimientos históricos y arqueológicos de la época. A la vista de lo que he venido señalando, me inclino a pensar que esa arbitrariedad es deliberada: dejándose llevar por el torbellino onomástico que él mismo ha generado, el poeta mezcla fechas y lugares, sin preocupación alguna por los datos históricos y ni siquiera por la coherencia interna del texto.

\section{DíAZ TANCO DE FREJENAL}

La hipótesis que he avanzado en las páginas anteriores puede verse reforzada por otro texto poético, de tema semejante al del romance, e indudablemente inspirado en la tradición de los mirabilia. Según confesión propia, el humanista Vasco Díaz Tanco de Frejenal se encontraba en Roma en 1527, y allí asistió al saqueo de la ciudad por parte de las tropas imperiales. Poco después, hacia 1530, dejó constancia de los hechos en un indigesto poema, el Triumpho púgnico lamentable, escrito en versos de arte mayor (Vian Herrero, 1994: 163-168).

Al igual que Juan de Mena, el autor construye muchas de sus estrofas mediante la anáfora del indefinido "vi", o "allí vi", al que sigue una enumeración de nombres propios prestigiosos, a veces sucintamente comentados. Versos como (Vian Herrero, 1994: 166; Mena, 1990: 220, copla 39): 
Allí vi la puente Theodosiana, con la que fue hecha por los senadores [...]

Allí vi muy triste el monte Auentino, y el otro de Jano con el Quirial [...]

parecen calcados, rítmica y estructuralmente, sobre los del Laberinto, como:

$\mathrm{Vi}$, de la parte que el Noto se enciende,

el Cáucaso monte cómo se levanta. [...]

Vi luego los Montes Iperboreos

Armenia e Scita con toda Albanía [...].

Pero a los recuerdos del Laberinto de Fortuna se yuxtapone el de las guías de Roma. Díaz Tanco presenta los monumentos no de acuerdo con las exigencias de la narración, sino agrupándolos en grandes categorías, que es lo que hacen casi siempre los mirabilia medievales y los textos humanísticos. El extremeño antepone a sus estrofas unos epígrafes que indican al lector qué tipo de edificios o de accidentes topográficos se tratan en la estrofa que sigue, que enlaza luego, no sin torpeza, con el relato de los hechos:
PUERTAS
Allí vi la puerta Capena y Latina, y la Methiona, Flaminea, Asinaria, y la Lanicaua, también la Salaria y la Numentana con la Tiburtina. [...] Las puertas ya dichas eran ocupadas de muertos y biuos con gran confusión.

Estas enumeraciones de monumentos, precedidas por un rótulo que los identifica claramente, son una herencia de las guías. Lo acaba de confirmar la lista de los epígrafes de Díaz Tanco:

1. Puertas (copla 13)

2. Puentes (c. 15)

3. Montes (c. 16)

4. Palacios (c. 17)

5. Theatros (c. 18)

6. Arcos triumphales (c. 19)

7. Arcos memoriales (c. 20)

8. Thermas (c. 22)

9. Templos (c. 24)

10. Capitolio (c. 26)

11. Coliseo (c. 27)

12. Aguja (c. 30) 
Pueden compararse esos epígrafes con los capítulos de uno de los mirabilia (Mirabilia Urbis Romae, 1510):

1. De portis infra Urbem

1. De portis Transtyberim

3. De montibus infra Urbem

2. De pontibus Urbis

4. Palatia imperatorum sunt hec

6. De arcubus triumphalibus

7. De arcubus non triumphalibus

8. De termis

5. De theatris

12. De Augulea Sancti Petri

- De cimiteriis

- Loca ubi sancti passi sunt

9. De templis

10. De Capitolio

- De equis marmoreis

- De rustico sedente super equum

11. De Coliseo

- De Sancta Maria Rotunda

- De Octaviano Imperatore

Las diferencias son notables e indican que no es este texto concreto el que sirve de base al poema. Pero las semejanzas también saltan a la vista, y muestran que ambas obras enlazan con la misma tradición. No sólo los apartados coinciden, sino que, en algunas secciones, su orden es idéntico. Así, se empieza por los cuatro apartados puertas — puentes-montes o montes-puentes - palacios, y se termina por la enumeración de monumentos concretos, es decir, de aquellos que merecen un tratamiento independiente. Es cierto que, en la guía, la aguja de San Pedro se cita antes, probablemente atraída por el contexto religioso de los cementerios y los escenarios de la muerte de los mártires. Pero la intención de reservar para el final los edificios de mayor fama y belleza es clara en ambos textos. Incluso se repite la secuencia "templos (último de los apartados generales) Capitolio-Coliseo". Algunos detalles son reveladores. Así, la división de los arcos en dos apartados diferentes: "De arcubus triumphalibus-De arcubus non triumphalibus; Arcos triunfales-Arcos memoriales". De hecho, en el cuerpo del texto latino, a los non triumphales se los llama también memoriales.

Lo que es casi indudable para el humanista extremeño me parece también muy probable para el anónimo autor del romance. Enfrentados a la destrucción de la ciudad, ambos poetas recurrieron a los textos que tenían más a mano para 
evocar la grandeza de lo que se estaba destruyendo. Quizá ambos actuaron de manera independiente, o quizá — como me inclino a creer- el humanista recurrió a los mirabilia bajo la sugestión del romance. Los contemporáneos asociaban el Saco de Roma al incendio del 64 d. C. (Díaz-Mas, 1994: 394-396), y los versos de "Mira Nero de Tarpeya" tuvieron que pasarle por la memoria a Díaz Tanco cuando se puso a escribir su composición. En cualquier caso, su poema explota de manera más torpe (y, por eso mismo, más evidente) las mismas fuentes que, con mayor discreción y acierto estético, parece haber utilizado también el autor del romance.

\section{BIBLIOGRAFÍA}

Alonso, Álvaro (2014): "Roma pagana y Roma cristiana: de Nerón al Saco de Roma en la poesía española”, en Luisa Secchi Tarugi (ed.), Roma pagana e Roma cristiana nel Rinascimento, Florencia, Franco Cesati, pp. 75-86.

Anderson, Gunnar (ed.) (1992): La corónica de Adramón, 2 vols., Newark, Juan de la Cuesta.

Biondo, Flavio (2005-2012): Rome restaurée. Roma instaurata, 2 vols., Anne Raffarin-Dupuis (ed. y trad.), Paris, Les Belles Lettres.

Bracciolini, Poggio (1993): De varietate Fortunae, Outi Merisalo (ed.), Helsinki, Soumalainen Tiedeakatemia.

Breyderbach, Bernardo de (1498): Viaje de la Tierra Santa, Martín Martínez de Ampiés (trad.), Zaragoza, Pablo Hurus.

Breyderbach, Bernardo de (2003 [1498]): Viaje de la Tierra Santa, Pedro Tena Tena (ed.), Zaragoza, Instituto Fernando el Católico.

Di Camillo, Ottavio (2010): "When and Where was the First Act of La Celestina Composed? A Reconsideration", en Devid Paolini (coord.), 'De ninguna cosa es alegre posesión sin compañía'. Estudios celestinescos y medievales en honor del profesor Joseph Thomas Snow, 2 vols., New York, Seminary of Hispanic Medieval Studies.

Di Stefano, Giuseppe (ed.) (1973): Romancero, Madrid, Ediciones Narcea.

Di Stefano, Giuseppe (ed.) (1993): Romancero, Madrid, Taurus.

Díaz-Mas, Paloma (1985): "Sobre la fortuna del romance "Mira Nero de Tarpeya"”, en José L. Melena (coord.), Symbolae Ludovico Mitxelena septuagenario oblatae, Vitoria, Diputación Foral de Álava.

Díaz-Mas, Paloma (ed.) (1994): Romancero, estudio preliminar de Samuel Armistead, Barcelona, Crítica.

Durán, Agustín (1945): Romancero general o colección de romances castellanos anteriores al siglo XVIII, tomo primero, Madrid, Atlas.

Fernández de Oviedo, Gonzalo (1989): Batallas y quinquagenas, Juan Bautista de Avalle-Arce (ed.), Salamanca, Ediciones de la Diputación de Salamanca.

García de Enterría, María Cruz (ed.) (1987): Romancero viejo (Antología), Madrid, Castalia.

Gómez Moreno, Ángel (1994): España y la Italia de los humanistas. Primeros ecos, Madrid, Gredos.

Leto, Pomponio (1521): De antiquitatibus urbis Romae, en Opera [...], Maguncia, Jacobus Ruyzius.

Mena, Juan de (1990): Laberinto de Fortuna, Miguel Ángel Pérez Priego (ed.), Barcelona, Planeta. Menéndez Pidal, Gonzalo (ed. y estudio preliminar) (1960): Romancero español, Barcelona, Éxito. 
Menéndez Pidal, Ramón (ed. e intr.) (1945): Cancionero de romances impreso en Amberes sin año, ed. facsímil, Madrid, CSIC.

Menéndez Pidal, Ramón (pról.) (1960): Pliegos poéticos españoles en la Universidad de Praga, II, Madrid, Joyas Bibliográficas.

Mirabilia Urbis Romae (1510): Marcellus Siber, Roma.

Murray, Peter (1972): Five Early Guides to Rome and Florence, Farnborough, Gregg International Publishers.

Pazienti, Massimo (2013): Le guide di Roma tra Medioevo e Novecento. Dai Mirabilia Urbis ai Baedaker, Roma, Gangemi editore.

Real Academia Española: Banco de datos (CORDE) [en línea]. Corpus diacrónico del español. $<$ http://corpus.rae.es/cordenet.html> [fecha de consulta: 28-6-2013].

Rodríguez-Moñino, Antonio (ed.) (1951): Cancionero gótico de Velázquez de Ávila, Valencia, Castalia.

Rodríguez-Moñino, Antonio (ed.) (1970): Silva de romances (Zaragoza, 1550-1551), Zaragoza, Publicaciones de la Cátedra Zaragoza.

Suetonio (1932): Vies des douze Césars, vol. 2, Tibère, Caligula, Claude, Néron, edición y traducción Henri Ailloud, Paris, Les Belles Lettres.

Tácito (1925): Annales. Livres XIII-XVI, edición y traducción Henri Goelzer, Paris, Les Belles Lettres.

Tácito (1980): Annales, libros XI-XVI, traducción y notas de José L. Moralejo, Madrid, Gredos.

Tena Tena, Pedro (1999): "Roma en textos españoles de viajes medievales", Lemir, 3. <http:// parnaseo.uv.es/lemir/revista/revista3/tena/tena.htm>.

Tortelli, Giovanni (1495): De ortographia dictionum e Graecais tractarum, Venecia, Johannes Tacuinus.

Valentini, Roberto y Giuseppe Zucchetti (1953): Codice topografico della città di Roma, IV, Roma, Tipografia del Senato.

Vian Herrero, Ana (1994): El 'Diálogo de Lactancio y un arcidiano' de Alfonso de Valdés: obra de circunstancias y diálogo literario. Roma en el banquillo de Dios, Toulouse-Le Mirail, Presses Universitaires du Mirail.

Fecha de recepción: 20 de julio de 2013

Fecha de aceptación: 24 de febrero de 2014 